\title{
Commentary
}

\section{Making light work of early gastric cancer}

Photodynamic therapy (PDT) should win the beauty contest between the differing endoscopic treatments for malignant or premalignant gastrointestinal mucosa with ease. The name could have been dreamed up by an advertising agency and the technique is based on the elegant theoretical advantage of dual selectivity resulting from the combination of a designer drug and laser light. Throw in local oxygen radical induced cytotoxicity and several unique safety features and you have an unbeatable product. However, although there is increasing interest in the use of PDT in gastroenterology, it is not new and remains experimental.

The initial step in PDT is the administration of a photosensitiser, most of which have been based on porphyrins. The photosensitiser is inert until activated by light, when localised cytotoxicity is produced-due to the production of oxygen radicals with most agents that have been used. An additional property of several photosensitisers has been their preferential retention in different tissues, particularly mucosa or neoplasms. By endoscopy most of the lesions suitable for PDT can be reached through the intestinal lumen and laser light directed at the abnormal area with sparing of adjacent normal mucosa. PDT possesses an important advantage over other techniques of tissue ablation, especially thermal destruction, in that while there is cytotoxicity and tissue necrosis, collagen remains intact, ${ }^{1}$ and hence the risks of perforation are reduced. ${ }^{2}$ Sunlight will also activate photosensitisers causing skin photosensitivity which has been the principal side effect of treatment and patients need to avoid natural light until the drug has been cleared. The other disadvantage of PDT is the limited penetration of light into tissue which restricts treatment to a depth of $10 \mathrm{~mm}$ although laser fibres can be inserted into the stroma to treat larger tumours.

In gastroenterology PDT has been used in three settings: premalignant change (Barrett's oesophagus); early malignancy (oesophagus, stomach, ampulla of Vater, and large intestine); and palliation of advanced malignancy (oesophagus, pancreas, cholangiocarcinoma, and large intestine). Few tumours incite more controversy than early gastric cancer (EGC) in terms of frequency, prognosis, need for screening, relation to advanced gastric cancer (AGC), and treatment.

Ell and coworkers have been among the main enthusiasts for PDT in Europe and in this issue (see page 345) describe the treatment of 22 patients with EGC by PDT using a second generation photosensitiser and achieving remission in $16(73 \%)$ with only minor complications. This is the first description of PDT for this indication in a series of patients who would otherwise have been treated conventionally, usually by surgery. Apart from the impressive results the study is one of the few from the West in a literature on EGC dominated by Japan. Although demographic data are not provided, the paper shows that EGC can be diagnosed and treated endoscopically in Europe with results similar to the Japanese experience of the disease. The study shows that PDT for EGC is feasible, safe and effective, but what clinical role will it have?

For the Western world the main limitation is the small number of patients in whom EGC is diagnosed. As a result of widespread screening in Japan more than half of all cases of gastric cancer are EGC (uT1). ${ }^{3}$ Despite the ever increasing numbers of patients having endoscopy in the UK cases of EGC are a minority even in units with an active programme for early diagnosis. ${ }^{4}$ Most Western gastroenterologists see few cases of EGC.

Surgery with its associated morbidity and mortality was avoided for the majority of the patients treated by Ell et al, but they had careful endoscopic and histological follow up. Although further work will be required to determine the optimum frequency and duration of follow up, several of the patients were relatively young and prolonged follow up, on at least an annual basis, may be required. For these patients a once and for all surgical cure will probably remain the most attractive option. On the other hand most of the patients were elderly with co-morbidity and would be suitable for PDT or alternative endoscopic treatment rather than surgery. Quite apart from the improved prognosis of EGC compared with AGC, elderly patients are unlikely to require such active or prolonged follow up. However, the drive for early endoscopic diagnosis of malignancy in the West is directed at younger patients, and the total number of cases of EGC in elderly or unfit patients may remain small.

Even if endoscopic therapy has a place in the treatment of EGC in selected cases, there are a number of techniques competing with PDT. Foremost is endoscopic mucosal resection (EMR) and its various modifications which are preferred in the literature from Japan. ${ }^{5}$ EMR does not require expensive or sophisticated equipment, is usually simple to perform, is relatively safe, and yields a complete resection specimen for histology. It could be argued that there is no place for treatment by ablation which includes laser, PDT, electrocoagulation, or alcohol injection. However, EMR has a complication rate of $1-18 \%$, with perforation of the stomach occurring in $8 \%$ of cases. ${ }^{5}$ In addition, there is a limit to the amount of mucosa that can be removed safely by EMR with a maximum diameter of around $25 \mathrm{~mm} .^{6}$ There is no such size restriction for ablative techniques and five of the 22 cases of EGC reported by Ell et al were at least $25 \mathrm{~mm}$ in diameter. Therefore PDT may be more suitable than EMR for larger lesions. EGC has been treated by Nd:YAG laser both in Japan and Europe with similar response rates and safety to PDT. ${ }^{7}$ The absence of skin phototoxicity with $\mathrm{Nd}$ :YAG laser treatment is countered by the need for multiple treatment sessions to eradicate EGC. Thus Sibille et al treated their 18 patients unfit for surgery with EGC less than $25 \mathrm{~mm}$ in diameter a median of four times (range $1-15) .{ }^{8}$ This difference is probably due to uneven thermal ablation with $\mathrm{Nd}$ :YAG laser treatment compared with even dosimetry to a field with PDT applied via the microlens developed by Ell et al. The need for multiple treatment sessions with $\mathrm{Nd}$ :YAG laser treatment is likely to be more of a problem for larger lesions and again PDT may be more suitable for such cases. 
Although there are these relatively minor differences in efficacy between the various endoscopic options for EGC, most of the few cases seen in the West could be satisfactorily treated by any of them if surgery is deemed inadvisable. The major differences are in the cost. The capital costs for EMR and for alcohol injection are minimal, for electrocoagulation $(£ 8000-20000$ (\$13 040$32600)$ ) moderate, for PDT by diode laser ( $£ 30000$ $(\$ 48900))$ and for Nd:YAG laser $(£ 40000$ (\$65 200)) expensive, and for PDT by tunable dye laser $(£ 100000$ (\$163 000)) very expensive. Diode lasers can only emit light of one preset wavelength which limits the choice of photosensitiser for PDT but as its name suggests a tunable dye laser is adjustable over a range of wavelengths and therefore can be used with a variety of photosensitisers. The scarcity of EGC not suitable for surgery in the West is such that for economic reasons treatment options are likely to be determined by either the existing availability of one of these competing techniques or if new equipment is being acquired the number of other clinical applications.

The study also emphasises the importance of assessment by endoscopic ultrasound (EUS). Although EUS is becoming established in most Western countries, less than 20 centres in the UK are equipped, and most patients will not have access to the technique. The number of centres able to offer both EUS and PDT is even smaller and it is ironic that this "dual" scarcity will limit the number of patients with EGC able to be treated by PDT.

However, there are good grounds for anticipating an increasing role for PDT in gastroenterology in general and for EGC in particular. With earlier diagnosis the number of cases of EGC in the West is likely to rise and a proportion of cases will be unsuitable for surgery. Third generation photosensitisers with improved selectivity and pharmacokinetics are already being evaluated and laser technology is both advancing and becoming cheaper. Although at present PDT remains experimental and restricted to a few centres, it has the potential to become the treatment of choice for a number of applications in the gastrointestinal tract.

Gastrointestinal Research Unit,

C AINLEY

St Bartholomerw's and the Royal London School of Medicine and Dentistry, 26 Ashfield Street, London E1 2A, UK

1 Barr H, Chantlani P, Tralau CJ, et al. Local eradication of rat colon cancer with PDT: correlation of distribution of photosensitizer with biological effects in normal and tumour tissue. Gut 1991;32:517-23.

2 Hisamichi S, Sugawara N. Mass screening for gastric cancer by x-ray examination. fpn f Clin Oncol 1984;14:211-15.

3 Sue-Ling HM, Martin I, Griffith J, et al. Early gastric cancer: 46 cases in one surgical department. Gut 1992;33:1318-22.

4 Lightdale CJ, Heier SK, Marcon NE, et al. Photodynamic therapy with porfimer sodium versus thermal ablation with Nd:YAG laser for palliation of oesophageal cancer: a multicenter randomized trial. Gastrointest Endosc 1995;42:507-12.

5 Hirao M, Masuda K, Asanuma T, et al. Endoscopic resection of early gastric cancer and other tumours with local injection of hypertonic salineepinephrine. Gastrointest Endosc 1988;34:264-9.

6 Karita $M$, Tada $M$, Okita $\mathrm{K}$. The successful strip biopsy, partial resection technique for early gastric and colon cancers. Gastrointest Endosc 1992;38: 174-8.

7 Takemoto T. Laser therapy of early gastric carcinoma. Endoscopy 1986;18(suppl 1):32-6.

8 Sibille A, Descamps C, Jonard P, et al. Endoscopic Nd:YAG treatment of superficial gastric carcinoma: experience in 18 Western inoperable patients. Gastrointest Endosc 1995;42:340-5.
It is unusual for a manuscript with negative conclusions to be highlighted in a commentary. However, the study by Reinisch et al (see page 375) is the latest of a number of reports to comment on the ability of an immunohistochemical stain for CD44 variant expression to discriminate between ulcerative colitis and Crohn's disease. It is a thorough study which puts earlier work into perspective, and as such is of considerable value.

The subject of Reinisch et al's study is epithelial expression of CD44, a receptor for matrix components including hyaluronic acid, which exists in multiple isoforms generated by exon splicing. ${ }^{12}$ The tissue distribution of CD 44 isoforms in normal and malignant tissues was first described in 1994 by Fox et al who observed weak expression of CD44v3 and CD44v6 isoforms on crypt epithelium in the gastrointestinal tract. ${ }^{3}$ Subsequently, Rosenberg et $a l,{ }^{4}$ using archival paraffin wax embedded specimens and a microwave system to reveal the epitopes recognised by the antibodies, observed a noticeable increase in the expression of CD44v3 and CD44v6 on crypt epithelium in ulcerative colitis compared with normal colon and colonic Crohn's disease. They proposed that this difference could form the basis of a diagnostic test to discriminate between these diseases. Such a test would clearly be of enormous value as the histopathologists' contribution to the disease classification, which continues to rely on a panel of classic histopathologi- cal features, sometimes fails to reach a satisfactory conclusion. Despite the resemblance between ulcerative colitis and Crohn's disease at many levels, they are presumed to be different diseases in terms of their aetiology and pathogenesis. The clinical management required is different, and accurate diagnosis is of considerable importance. Rosenberg et al's study was confirmed by two other groups ${ }^{5}{ }^{6}$ but a third study ${ }^{7}$ failed to reproduce these results. The two groups which had agreed had used frozen sections, whereas the group which had failed to discriminate between ulcerative colitis and Crohn's disease had used paraffin wax sections. These studies all used the same antibodies.

The study by Reinisch et al concentrates on the expression of CD44v6. They first produced a high affinity antibody to CD44v6, which they studied alongside the $2 \mathrm{~F} 10$ anti-CD44v6 monoclonal used in previous studies. They then stained a panel of paraffin wax specimens using a microwave technique to reveal the antigens, and assessed the reactivity in terms of the intensity of expression and the number of positive cells. Like Rosenberg et al, they observed that CD44v6 expression was higher in ulcerative colitis than in Crohn's disease in both the intensity of the staining and the number of positive cells. Both antibodies gave the same pattern of staining though the higher affinity antibody stained more cells, and stained more intensely. These authors state, however, that the staining would not constitute a diagnostic test because of deficiencies in specificity and sensitivity. Reinisch et al therefore confirm both the original observation of an increase in CD44v6 staining in ulcerative colitis and also the doubts of others about the diagnostic value of the observation. 
The very fact that there was debate on the usefulness of CD44v6 staining stymied its prospects as a diagnostic test. In modern pathology laboratories, diagnostic immunohistochemical techniques are regularly carried out using paraffin wax sections. Different laboratories use different antigen retrieval systems to reveal epitopes masked by the processes of fixation and embedding. Alternative techniques are trypsinisation, microwave or pressure cooking. Though different antigens may be optimally revealed by different processes, selection of technique used is often based on personal experience. Variability in staining can depend on factors such as the local formalin (buffered or not) and the size of the piece of tissue (resection or biopsy). To achieve success as a diagnostic test, the antibody has to overcome these variables and bind to its target reliably and reproducibly. The failure of Papadogiannakis et al to observe any difference in CD44v6 expression between ulcerative colitis and Crohn's disease could have been due to any of these variables.

From the pathologist's point of view, the most difficult immunohistochemical reactivity to assess must be that which depends on degree of positivity. When Rosenberg et al's paper was first published, ${ }^{4}$ Ilyas and colleagues ${ }^{8}$ pointed out to the authors that CD44v6 did in fact stain normal colonic crypt epithelium, ${ }^{38}$ albeit weakly, a point which was dismissed by Rosenberg as possibly semantic. ${ }^{9}$ This weak staining may well have been easy for those who see many such specimens stained in the same way to ignore, but to a diagnostic pathologist, it would be important to understand that weakly positive staining might be a negative result. CD44v6 staining would have to be examined alongside specimens with high intensity and weak staining, and the shade of the positivity considered in this context. Failure of a specimen to stain using an immunohistochemical technique, with all of the variables which can affect staining mentioned earlier, is not interpretable. In addition, epithelial cells frequently show weak nonspecific background staining; a fact which could add to the confusion.

In conclusion, although scientifically the increase in CD44v6 expression in ulcerative colitis appears to be a sound and interesting observation, the fact that it relies on a differential expression over a variable background makes it useless for routine diagnosis. The differential diagnosis of Crohn's disease and ulcerative colitis must remain a multidepartmental collaborative effort, at least for the time being.

J SPENCER

Department of Histopathology,

UMDS St Thomas's Campus,

Lambeth Palace Road,

London SE1 7EH, UK

1 Lesley J, Hyman R, Kincade PW. CD44 and its interaction with extracellular matrix. Adv Immunol 1993;54:271-335.

2 Henke CA, Roongta U, Mickelson DJ, et al. CD44-related chondroitin sulfate proteoglycan, a cell surface receptor implicated with tumour cell invasion, mediates endothelial cell migration an fibrin and invasion into a fibrin matrix. F Clin Invest 1996;97:2541-52.

3 Fox SB, Fawcett J, Jackson DG, et al. Normal human tissues, in addition to some tumors, express multiple different CD44 isoforms. Cancer Res 1994; 54:4539-46.

4 Rosenberg WMC, Prince C, Laklamanis L, et al. Increased expression of CD $44 \mathrm{v} 6$ and CD $44 \mathrm{v} 3$ in ulcerative colitis but not Crohn's disease. Lancet CD44v6 and CD

5 Cellier C, Patey N, Leborgne M, et al. In situ expression of CD44 in ulcerative colitis (UC) and colonic Crohn's disease (CD) [abstract]. Gastroenterology 1996;110:A880.

6 Kitano A, Oshitani N, Matsumoto T, et al. CD44 variants in ulcerative colitis and Crohn's disease. Lancet 1996;348:266-7.

7 Papadogiannakis N, Gad A, Chenard M-P, et al. Expression of CD44 variants in differential diagnosis of ulcerative colitis and Crohn's disease. Lancet 1996;347:1413-14.

8 Ilyas M, Abbasi, AM, Talbot IC, et al. CD44v6 and CD44v3 expression in ulcerative coltitis and Crohn's disease [letter]. Lancet 1995;346:256.

9 Rosenberg W. CD44v6 and CD44v3 expression in ulcerative coltitis and Crohn's disease [reply]. Lancet 1995;346:256. 